
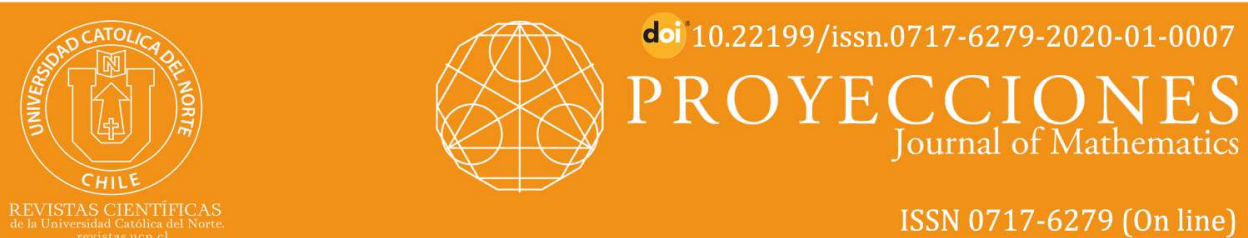

\title{
General solution and hyperstability results for a cubic radical functional equation related to quadratic mapping
}

Rachid El Ghali ${ }^{1}$

Muaadh Almahalebi ${ }^{2}$ @ orcid.org/0000-0003-0647-8286

Samir Kabbaj ${ }^{3}$ ๑ orcid.org/0000-0001-9717-9366

Ibn Tofail University, Dept. of Mathematics, Kenitra, Morocco.

1凶rachid2810@gmail.com; 2 muaadh1979@hotmail.fr; $3{ }_{\text {samkabbaj@yahoo.fr }}$

Received: December 2018 | Accepted: June 2019

\section{Abstract:}

The aim of this paper is to introduce and solve the following radical cubic functional equation

$$
f\left(\sqrt[3]{x^{3}+y^{3}}\right)+f\left(\sqrt[3]{x^{3}-y^{3}}\right)=2 f(x)+2 f(y), x, y \in \mathbf{R}
$$

Also, we investigate some stability results for the considered equation in Banach spaces.

Keywords: Stability; Hyperstability; Radical functional equations.

MSC (2010): 39B82; 39B62, 47H14, 47J20, 47H10.

\section{Cite this article as (IEEE citation style):}

R. El Ghali, M. Almahalebi and S. Kabbaj, "General solution and hyperstability results for a cubic radical functional equation related to quadratic mapping", Proyecciones (Antofagasta, On line), vol. 39, no. 1, pp. 107-122, Feb. 2020, doi: 10.22199/issn.07176279-2020-01-0007. [Accessed dd-mm-yyyy].

Article copyright: (C) 2020 Rachid El Ghali, Muaadh Almahalebi and Samir Kabbaj. This is an open access article distributed under the terms of the Creative Commons Licence, which permits unrestricted use and distribution provided the original author and source are credited.

(cc) BY 


\section{Introduction}

If we define, in some way, the class of approximate solutions of given functional equation, then the natural question arises in many areas of scientific investigation is what errors we commit replacing each function in this class by the exact solution of that equation.

The evaluation of those errors can be found in the theory of Ulam's stability, see for example $([3,4,5,10,14,15,16,17])$.

When the approximate of a functional equation is a true solution of it, we say that this equation is hyperstable. The term hyperstability was used for the first time probably in [13]. However, the hyperstability result for the Cauchy equation were investigated by Brzdȩk in [7] and other results of hyperstability of functional equations have been studied by many authors, for example, $([1,2,7,8,10,11])$.

Throughout this paper, we will denote $X$ the Banach space, the set of natural numbers by $\mathbf{N}$, the set of real numbers by $\mathbf{R}$ and $\mathbf{R}_{+}=[0, \infty)$ the set of non-negative real numbers. By $\mathbf{N}_{m_{0}}, m_{0} \in \mathbf{N}$, we will denote the set of all natural numbers greater than or equal to $m_{0}$. We say that a function $f: \mathbf{R} \longrightarrow X$ satisfies the radical cubic functional equation related to quadratic mapping if :

$$
f\left(\sqrt[3]{x^{3}+y^{3}}\right)+f\left(\sqrt[3]{x^{3}-y^{3}}\right)=2 f(x)+2 f(y)
$$

for all $x, y \in \mathbf{R}$. Indeed, there are many authors who studied the radical functional equation. See, for example, $([8,9,11,12])$.

In this paper, we show the solution of the radical cubic functional equation on the set of the real numbers $\mathbf{R}$, and we will also prove the hyperstability of the equation (1.1), that is, for each function $f: \mathbf{R} \longrightarrow X$ satisfying the inequality:

$$
\left\|f\left(\sqrt[3]{x^{3}+y^{3}}\right)+f\left(\sqrt[3]{x^{3}-y^{3}}\right)-2 f(x)-2 f(y)\right\| \leq c\left(|x|^{p}+|y|^{p}\right)
$$

or

$$
\left\|f\left(\sqrt[3]{x^{3}+y^{3}}\right)+f\left(\sqrt[3]{x^{3}-y^{3}}\right)-2 f(x)-2 f(y)\right\| \leq c\left(|x|^{p} \cdot|y|^{q}\right)
$$


for all $x, y \in \mathbf{R} \backslash\{0\}$ such that $x \neq y$ and $x \neq-y$ must satisfy the radical cubic functional equation related to the quadratic mapping.

The method of the proof of the main theorem is motivated by an idea used by J.Brzdęk in [7].

We need to present the basic tool of our results that given in [6]. First, we recall the following three hypotheses, all notation come from [6].

$X$ is a Banach space and $Y$ is a nonempty set.

$\left(H_{1}\right): f_{1}, \ldots, f_{k}: Y \longrightarrow Y L_{1}, \ldots, L_{k}: Y \longrightarrow \mathbf{R}_{+}$are given.

$\left(H_{2}\right): \mathcal{T}: X^{Y} \longrightarrow X^{Y}$ is an operator satisfying the inequality :

$$
\|\mathcal{T} \xi(x)-\mathcal{T} \mu(x)\| \leq \sum_{i=1}^{k} L_{i}(x) \| \xi\left(f_{i}(x)\right)-\mu\left(f_{i}(x) \|, \quad \xi, \mu \in X^{Y}, x \in Y .\right.
$$

$\left(H_{3}\right): \Lambda: \mathbf{R}_{+}{ }^{Y} \longrightarrow \mathbf{R}_{+}{ }^{Y}$ is defined by :

$$
\Lambda \delta(x)=\sum_{i=1}^{k} L_{i}(x) . \delta\left(f_{i}(x)\right), \quad \delta \in \mathbf{R}_{+}{ }^{Y}, x \in Y .
$$

Theorem 1.1. [6] $Y$ is a nonempty set and $X$ is Banach space. Let hypotheses are valid and functions $\varepsilon: Y \longrightarrow \mathbf{R}_{+}$and $\varphi: Y \longrightarrow X$ fulfil the following two conditions:

$$
\begin{aligned}
& \|\mathcal{T} \varphi(x)-\varphi(x)\| \leq \varepsilon(x) \quad, x \in Y . \\
& \varepsilon^{*}(x)=\sum_{n=0}^{\infty} \Lambda^{n} \varepsilon(x)<\infty \quad, x \in Y .
\end{aligned}
$$

Then, there exists a unique fixed point $\psi$ of $\mathcal{T}$ with

$$
\|\varphi(x)-\psi(x)\| \leq \varepsilon^{*}(x) \quad, x \in Y .
$$

Moreover

$$
\psi(x)=\lim _{n \rightarrow \infty} \mathcal{T}^{n} \varphi(x) \quad, x \in Y
$$




\section{The main result}

In this section, we give the general solution of functional equation (1.1). The proof of the the following theorem has been patterned on the reasoning in [8].

Theorem 2.1. Let $X$ be a Banach space. A function $f: \mathbf{R} \rightarrow X$ satisfies the functional equation (1.1) if and only if $f(x)=F\left(x^{3}\right)$, with some quadratic function $F: \mathbf{R} \rightarrow X$.

Proof. Indeed, if $f(x)=F\left(x^{3}\right)$ for all $x \in \mathbf{R}$, with $F$ is a quadratic function.

Then, for all $x, y \in \mathbf{R}$ :

$$
\begin{aligned}
f\left(\sqrt[3]{x^{3}+y^{3}}\right)+f\left(\sqrt[3]{x^{3}-y^{3}}\right) & =F\left(x^{3}+y^{3}\right)+F\left(x^{3}-y^{3}\right) \\
& =2 F\left(x^{3}\right)+2 F\left(y^{3}\right) \\
& =2 f(x)+2 f(y)
\end{aligned}
$$

Then $f$ is a solution of (1.1).

On the other hand, if $f: \mathbf{R} \rightarrow X$ is a solution of (1.1), then we write $F(x)=f(\sqrt[3]{x})$ for $x \in \mathbf{R}$, we obtain that :

$$
\begin{aligned}
F(x+y)+F(x-y) & =f(\sqrt[3]{x+y})+f(\sqrt[3]{x-y}) \\
& =2 f(\sqrt[3]{x})+2 f(\sqrt[3]{y}) \\
& =2 F(x)+2 F(y)
\end{aligned}
$$

for all $x, y \in \mathbf{R}$.

Theorem 2.2. Let $X$ be a Banach space, $c \geq 0$ and $p<0$. If $f: \mathbf{R} \rightarrow X$ satisfies :

$$
\begin{gathered}
\left\|f\left(\sqrt[3]{x^{3}+y^{3}}\right)+f\left(\sqrt[3]{x^{3}-y^{3}}\right)-2 f(x)-2 f(y)\right\| \\
\leq c\left(|x|^{p}+|y|^{p}\right), x, y \in \mathbf{R} \backslash\{0\}, x \neq y, x \neq-y .
\end{gathered}
$$

Then $f$ satisfies the functional equation (1.1) on $\mathbf{R} \backslash\{0\}$.

Proof. First, observe that there exists $m_{0} \in \mathbf{N}_{0}$ such that :

$$
2 \sqrt[3]{m+1}^{p}+2 \sqrt[3]{m}^{p}+\sqrt[3]{2 m+1}^{p}<1, \quad m \geq m_{0}
$$

Let us fix $m \in \mathbf{N}_{m_{0}}$. Replacing $x$ by $\sqrt[3]{m+1} x$ and $y$ by $\sqrt[3]{-m} x$ in inequality (2.1), we get : 
$\|2 f(\sqrt[3]{m+1} x)+2 f(\sqrt[3]{-m} x)-f(\sqrt[3]{2 m+1} x)-f(x)\| \leq c\left(\sqrt[3]{m+1}^{p}+\sqrt[3]{m}^{p}\right)|x|^{p}$

for $x \in \mathbf{R} \backslash\{0\}$. Further, we define operators $\mathcal{T}: X^{\mathbf{R}} \rightarrow X^{\mathbf{R}}$ and $\varepsilon: \mathbf{R} \rightarrow$ $\mathbf{R}_{+}$by

$$
\mathcal{T} \xi(x)=2 \xi(\sqrt[3]{m+1} x)+2 \xi(\sqrt[3]{-m} x)-\xi(\sqrt[3]{2 m+1} x)
$$

for $x \in \mathbf{R} \backslash\{0\}, \xi \in X^{\mathbf{R}}$, and

$$
\varepsilon(x)=c\left(\sqrt[3]{m+1}^{p}+\sqrt[3]{m}^{p}\right)|x|^{p}, \quad x \in \mathbf{R} \backslash\{0\} .
$$

Inequality (2.2) become as the form :

$$
\|\mathcal{T} f(x)-f(x)\| \leq \varepsilon(x), x \in \mathbf{R} \backslash\{0\} .
$$

The operator :

$\Lambda \eta(x)=2 \eta(\sqrt[3]{m+1} x)+2 \eta(\sqrt[3]{-m} x)+\eta(\sqrt[3]{2 m+1} x), \quad \eta \in \mathbf{R}_{+}^{\mathbf{R}}, x \in \mathbf{R} \backslash\{0\}$ has the form described in $\left(H_{3}\right)$ with $k=3$ and

$f_{1}(x)=\sqrt[3]{m+1} x, f_{2}(x)=\sqrt[3]{-m} x, f_{3}(x)=\sqrt[3]{2 m+1} x$ and $L_{1}(x)=2, L_{2}(x)=2, L_{3}(x)=1$.

Moreover, for every $\xi, \mu \in X^{\mathbf{R} \backslash\{0\}}, x \in \mathbf{R} \backslash\{0\}$ :

$$
\begin{aligned}
\|\mathcal{T} \xi(x)-\mathcal{T} \mu(x)\| & =\| 2(\xi(\sqrt[3]{m+1} x)-\mu(\sqrt[3]{m+1} x))+2(\xi(\sqrt[3]{-m} x)-\mu(\sqrt[3]{-m} x)) \\
& -(\xi(\sqrt[3]{2 m+1} x)-\mu(\sqrt[3]{2 m+1} x)) \| \\
& \leq 2\|(\xi-\mu)(\sqrt[3]{m+1} x)\|+2\|(\xi-\mu)(\sqrt[3]{-m} x)\|+\|(\xi-\mu)(\sqrt[3]{2 m+1} x)\| \\
& =\sum_{i=1}^{3} L_{i}(x)\left\|(\xi-\mu) f_{i}(x)\right\|
\end{aligned}
$$

As $2 \sqrt[3]{m+1}^{p}+2 \sqrt[3]{m}^{p}+\sqrt[3]{2 m+1}^{p}<1$, we have :

$$
\begin{aligned}
& \varepsilon^{*}(x)=\sum_{n=0}^{\infty} \Lambda^{n} \varepsilon(x) \\
& =\sum_{n=0}^{\infty} \Lambda^{n}\left(c\left(\sqrt[3]{m+1} p+\sqrt[3]{m^{p}}\right)|x|^{p}\right)
\end{aligned}
$$$$
=c\left(\sqrt[3]{m+1}^{p}+\sqrt[3]{m^{p}}\right) \sum_{n=0}^{\infty}\left(2 \sqrt[3]{m+1}^{p}+2 \sqrt[3]{m}^{p}+\sqrt[3]{2 m+1} p\right)^{n}|x|^{p}
$$$$
=\frac{c\left(\sqrt[3]{m+1}{ }^{p}+\sqrt[3]{m^{p}}\right)|x|^{p}}{1-\left(2 \sqrt[3]{m+1^{p}}+2 \sqrt[3]{m^{p}}+\sqrt[3]{2 m+1}\right)}<\infty
$$ 
Thus, according to theorem (1.1), there exists a fixed point $\mathcal{T}_{m}: \mathbf{R} \rightarrow X$ of the operator $\mathcal{T}$ such that:

$$
\left\|f(x)-\mathcal{T}_{m}(x)\right\| \leq \frac{c\left(\sqrt[3]{m+1} p+\sqrt[3]{m}^{p}\right)|x|^{p}}{1-\left(2 \sqrt[3]{m+1}^{p}+2 \sqrt[3]{m}^{p}+\sqrt[3]{2 m+1}^{p}\right)}
$$

Moreover $\mathcal{T}_{m}(x)=\lim _{n \rightarrow \infty} \mathcal{T}^{n}(f(x)), x \in \mathbf{R} \backslash\{0\}$. To prove that $\mathcal{T}_{m}$ is a solution of the radical cubic function equation, we will show that :

$$
\begin{gathered}
\left\|\mathcal{T}^{n} f\left(\sqrt[3]{x^{3}+y^{3}}\right)+\mathcal{T}^{n} f\left(\sqrt[3]{x^{3}-y^{3}}\right)-2 \mathcal{T}^{n} f(x)-2 \mathcal{T}^{n} f(y)\right\| \\
\leq c\left(2 \sqrt[3]{m+1}^{p}+2 \sqrt[3]{m}^{p}+\sqrt[3]{2 m+1}^{p}\right)^{n}\left(|x|^{p}+|y|^{p}\right)
\end{gathered}
$$

for $x, y \in \mathbf{R} \backslash\{0\}$ with $x \neq y, x \neq-y$ and $n \in \mathbf{N}_{0}$.

Indeed, if $n=0$, then (2.4) is simply (2.1). So we take $n \in \mathbf{N}_{0}$ and suppose that (2.4) holds for $n$ and $x, y \in \mathbf{R} \backslash\{0\}$ with $x \neq y$ and $x \neq-y$, then 


$$
\begin{aligned}
& \left\|\mathcal{T}^{n+1} f\left(\sqrt[3]{x^{3}+y^{3}}\right)+\mathcal{T}^{n+1} f\left(\sqrt[3]{x^{3}-y^{3}}\right)-2 \mathcal{T}^{n+1} f(x)-2 \mathcal{T}^{n+1} f(y)\right\| \\
& =\left\|\mathcal{T}\left(\mathcal{T}^{n} f\left(\sqrt[3]{x^{3}+y^{3}}\right)\right)+\mathcal{T}\left(\mathcal{T}^{n} f\left(\sqrt[3]{x^{3}-y^{3}}\right)\right)-2 \mathcal{T}\left(\mathcal{T}^{n} f(x)\right)-2 \mathcal{T}\left(\mathcal{T}^{n} f(y)\right)\right\| \\
& =\| 2 \mathcal{T}^{n} f\left(\sqrt[3]{m+1} \sqrt[3]{x^{3}+y^{3}}\right)+2 \mathcal{T}^{n} f\left(\sqrt[3]{-m} \sqrt[3]{x^{3}+y^{3}}\right)-\mathcal{T}^{n} f\left(\sqrt[3]{2 m+1} \sqrt[3]{x^{3}+y^{3}}\right) \\
& +2 \mathcal{T}^{n} f\left(\sqrt[3]{m+1} \sqrt[3]{x^{3}-y^{3}}\right)+2 \mathcal{T}^{n} f\left(\sqrt[3]{-m} \sqrt[3]{x^{3}-y^{3}}\right)-\mathcal{T}^{n} f\left(\sqrt[3]{2 m+1} \sqrt[3]{x^{3}-y^{3}}\right) \\
& -4 \mathcal{T}^{n} f(\sqrt[3]{m+1} x)-4 \mathcal{T}^{n} f(\sqrt[3]{-m} x)+2 \mathcal{T}^{n} f(\sqrt[3]{2 m+1} x) \\
& -4 \mathcal{T}^{n} f(\sqrt[3]{m+1} y)-4 \mathcal{T}^{n} f(\sqrt[3]{-m} y)+2 \mathcal{T}^{n} f(\sqrt[3]{2 m+1} y) \\
& =\| 2 \mathcal{T}^{n} f\left(\sqrt[3]{(\sqrt[3]{m+1} x)^{3}+(\sqrt[3]{m+1} y)^{3}}\right)+2 \mathcal{T}^{n} f\left(\sqrt[3]{(\sqrt[3]{-m} x)^{3}+(\sqrt[3]{-m} y)^{3}}\right) \\
& -\mathcal{T}^{n} f\left(\sqrt[3]{(\sqrt[3]{2 m+1} x)^{3}+(\sqrt[3]{2 m+1} y)^{3}}\right)+2 \mathcal{T}^{n} f\left(\sqrt[3]{(\sqrt[3]{m+1} x)^{3}-(\sqrt[3]{m+1} y)^{3}}\right) \\
& +2 \mathcal{T}^{n} f\left(\sqrt[3]{(\sqrt[3]{-m} x)^{3}-(\sqrt[3]{-m} y)^{3}}\right)-\mathcal{T}^{n} f\left(\sqrt[3]{(\sqrt[3]{2 m+1} x)^{3}-(\sqrt[3]{2 m+1} y)^{3}}\right) \\
& -4 \mathcal{T}^{n} f(\sqrt[3]{m+1} x)-4 \mathcal{T}^{n} f(\sqrt[3]{-m} x)+2 \mathcal{T}^{n} f(\sqrt[3]{2 m+1} x) \\
& -4 \mathcal{T}^{n} f(\sqrt[3]{m+1} y)-4 \mathcal{T}^{n} f(\sqrt[3]{-m} y)+2 \mathcal{T}^{n} f(\sqrt[3]{2 m+1} y) \\
& \leq 2 \| \mathcal{T}^{n} f\left(\sqrt[3]{(\sqrt[3]{m+1} x)^{3}+(\sqrt[3]{m+1} y)^{3}}\right)+\mathcal{T}^{n} f\left(\sqrt[3]{(\sqrt[3]{m+1} x)^{3}-(\sqrt[3]{m+1} y)^{3}}\right) \\
& -2 \mathcal{T}^{n} f(\sqrt[3]{m+1} x)-2 \mathcal{T}^{n} f(\sqrt[3]{m+1} y) \| \\
& +2 \| \mathcal{T}^{n} f\left(\sqrt[3]{(\sqrt[3]{-m} x)^{3}+(\sqrt[3]{-m} y)^{3}}\right)+\mathcal{T}^{n} f\left(\sqrt[3]{(\sqrt[3]{-m} x)^{3}-(\sqrt[3]{-m} y)^{3}}\right) \\
& -2 \mathcal{T}^{n} f(\sqrt[3]{-m} x)-2 \mathcal{T}^{n} f(\sqrt[3]{-m} y) \\
& +\| \mathcal{T}^{n} f\left(\sqrt[3]{(\sqrt[3]{2 m+1} x)^{3}+(\sqrt[3]{2 m+1} y)^{3}}\right)+\mathcal{T}^{n} f\left(\sqrt[3]{(\sqrt[3]{2 m+1} x)^{3}-(\sqrt[3]{2 m+1} y)^{3}}\right) \\
& -2 \mathcal{T}^{n} f(\sqrt[3]{2 m+1} x)-2 \mathcal{T}^{n} f(\sqrt[3]{2 m+1} y) \| \\
& \leq c\left(2 \sqrt[3]{m+1}^{p}+2{\sqrt[3]{m^{p}}}^{p}+\sqrt[3]{2 m+1}^{p}\right)^{n}\left(2 \sqrt[3]{m+1}^{p}\left(|x|^{p}+|y|^{p}\right)\right) \\
& +c\left(2 \sqrt[3]{m+1}^{p}+2 \sqrt[3]{m}^{p}+\sqrt[3]{2 m+1}^{p}\right)^{n}\left(2 \sqrt[3]{m}^{p}\left(|x|^{p}+|y|^{p}\right)\right) \\
& +c\left(2 \sqrt[3]{m+1}^{p}+2 \sqrt[3]{m}^{p}+\sqrt[3]{2 m+1}^{p}\right)^{n}\left(2 \sqrt[3]{2 m+1}^{p}\left(|x|^{p}+|y|^{p}\right)\right) \\
& \leq c\left(2 \sqrt[3]{m+1}^{p}+2 \sqrt[3]{m}^{p}+\sqrt[3]{2 m+1}^{p}\right)^{n+1}\left(|x|^{p}+|y|^{p}\right)
\end{aligned}
$$

By induction, we show that (2.4) holds for all $x, y \in \mathbf{R} \backslash\{0\}$ with $x \neq y$, $x \neq-y$ and $n \in \mathbf{N}_{0}$. Letting $n \rightarrow \infty$ in (2.4), we obtain :

$\mathcal{T}_{m}\left(\sqrt[3]{x^{3}+y^{3}}\right)+\mathcal{T}_{m}\left(\sqrt[3]{x^{3}-y^{3}}\right)=2 \mathcal{T}_{m}(x)+2 \mathcal{T}_{m}(y) \quad x, y \in \mathbf{R} \backslash\{0\}, x \neq-y, x \neq y$

We prove now that $\mathcal{T}_{m}$ is the unique operator solution of (1.1) and satisfies the condition (2.3) for all $x \in \mathbf{R} \backslash\{0\}$.

So, let $\mathcal{T}_{0}: \mathbf{R} \rightarrow X$ an other solution of (1.1) such that : 


$$
\left\|f(x)-\mathcal{T}_{0}(x)\right\| \leq \frac{c\left(\sqrt[3]{m+1}^{p}+\sqrt[3]{m}^{p}\right)|x|^{p}}{1-\left(2 \sqrt[3]{m+1}^{p}+2 \sqrt[3]{m}^{p}+\sqrt[3]{2 m+1}^{p}\right)} \quad, x \in \mathbf{R} \backslash\{0\}
$$

then for every $x \in \mathbf{R} \backslash\{0\}$ :

$$
\begin{gathered}
\left\|\mathcal{T}_{m}(x)-\mathcal{T}_{0}(x)\right\| \leq\left\|\mathcal{T}_{m}(x)-f(x)\right\|+\left\|\mathcal{T}_{0}(x)-f(x)\right\| \\
\leq \frac{2 c\left(\sqrt[3]{m+1}^{p}+\sqrt[3]{m}^{p}\right)|x|^{p}}{1-\left(2 \sqrt[3]{m+1}+2 \sqrt[3]{m}^{p}+\sqrt[3]{2 m+1}\right)}
\end{gathered}
$$

Putting

$$
\beta=2 c\left(\sqrt[3]{m+1}^{p}+\sqrt[3]{m}^{p}\right)
$$

and show that

$$
\left\|\mathcal{T}_{m}(x)-\mathcal{T}_{0}(x)\right\| \leq \beta|x|^{p} \sum_{n=j}^{n=\infty}\left(2 \sqrt[3]{m+1}^{p}+2 \sqrt[3]{m}^{p}+\sqrt[3]{2 m+1}^{p}\right)^{n}
$$

for each $j \in \mathbf{N}_{0}$ and $x \in \mathbf{R} \backslash\{0\}$.

For $j=0$ inequality (2.7) is exactly (2.6). So we fix $l \in \mathbf{N}_{0}$ and assume that (2.7) holds for $j=l$, then

$$
\begin{aligned}
& \left\|\mathcal{T}_{m}(x)-\mathcal{T}_{0}(x)\right\|=\| 2 \mathcal{T}_{m}(\sqrt[3]{m+1} x)+2 \mathcal{T}_{m}(\sqrt[3]{-m} x)-\mathcal{T}_{m}(\sqrt[3]{2 m+1} x) \\
& -2 \mathcal{T}_{0}(\sqrt[3]{m+1} x)-2 \mathcal{T}_{0}(\sqrt[3]{-m} x)+\mathcal{T}_{0}(\sqrt[3]{2 m+1} x) \| \\
& \leq 2\left\|\mathcal{T}_{m}(\sqrt[3]{m+1} x)-\mathcal{T}_{0}(\sqrt[3]{m+1} x)\right\|+2\left\|\mathcal{T}_{m}(\sqrt[3]{-m} x)-\mathcal{T}_{0}(\sqrt[3]{-m} x)\right\| \\
& +\left\|\mathcal{T}_{m}(\sqrt[3]{2 m+1} x)-\mathcal{T}_{0}(\sqrt[3]{2 m+1} x)\right\| \\
& \leq \beta\left(2 \sqrt[3]{m+1}^{p}+2 \sqrt[3]{m}^{p}+\sqrt[3]{2 m+1} p\right)|x|^{p} \sum_{n=l}^{n=\infty}\left(2 \sqrt[3]{m+1} p+2 \sqrt[3]{m}^{p}+\sqrt[3]{2 m+1}^{p}\right)^{n} \\
& =\beta|x|^{p} \sum_{n=l+1}^{n=\infty}\left(2 \sqrt[3]{m+1}+2 \sqrt[3]{m^{p}}+\sqrt[3]{2 m+1}\right)^{n}, \quad x \in \mathbf{R} \backslash\{0\}
\end{aligned}
$$


Thus, we have shown that (2.7) holds true for every $j \in \mathbf{N}_{0}$. Now letting $j \rightarrow \infty$ in $(2.7)$, we get $\mathcal{T}_{m}=\mathcal{T}_{0}$.

Theorem 2.3. Let $X$ be a Banach space, $c \geq 0$ and $p>6$. If $f: \mathbf{R} \rightarrow X$ satisfies (2.1). Then $f$ satisfies the radical cubic functional equation related to quadratic mapping on $\mathbf{R} \backslash\{0\}$.

Proof. By a similarly proof of the previous theorem with replacing $x$ by $\sqrt[3]{\frac{m+1}{2 m}} x$ and $y$ by $\sqrt[3]{\frac{m-1}{2 m}} x$ we can easily prove this one. Obviously, there exists $m_{0} \in \mathbf{N}_{0}$ such that

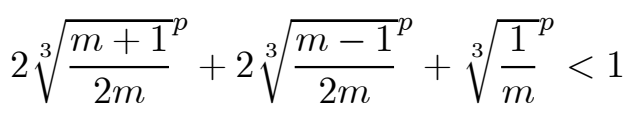

We define operators as following :

$$
\begin{aligned}
& \mathcal{T} \xi(x)=2 \xi\left(\sqrt[3]{\frac{m+1}{2 m}} x\right)+2 \xi\left(\sqrt[3]{\frac{m-1}{2 m}} x\right)-\xi\left(\sqrt[3]{\frac{1}{m}} x\right), \quad x \in \mathbf{R} \backslash\{0\}, \xi \in X^{\mathbf{R}} \\
& \varepsilon(x)=c\left(\sqrt[3]{\frac{m+1}{2 m}}^{p}+\sqrt[3]{\frac{m-1}{2 m}}^{p}\right)|x|^{p}, \quad x \in \mathbf{R} \backslash\{0\} \\
& \Lambda \eta(x)=2 \eta\left(\sqrt[3]{\frac{m+1}{2 m}} x\right)+2 \eta\left(\sqrt[3]{\frac{m-1}{2 m}} x\right)+\eta\left(\sqrt[3]{\frac{1}{m}} x\right), \quad x \in \mathbf{R} \backslash\{0\}, \eta \in \mathbf{R}_{+}^{\mathbf{R}} \\
& f_{1}(x)=\sqrt[3]{\frac{m+1}{2 m}} x, f_{2}(x)=\sqrt[3]{\frac{m-1}{2 m}} x, f_{3}(x)=\sqrt[3]{\frac{1}{m}} x \\
& L_{1}(x)=L_{2}(x)=2, L_{3}(x)=1
\end{aligned}
$$

Next, we investigate the hyperstability of equation (1.1) in an other way.

Theorem 2.4. Let $X$ be a Banach space, $c \geq 0$ and $p, q \in \mathbf{R}$ such that $p+q<0$.

If $f: \mathbf{R} \rightarrow X$ satisfies :

$$
\left\|f\left(\sqrt[3]{x^{3}+y^{3}}\right)+f\left(\sqrt[3]{x^{3}-y^{3}}\right)-2 f(x)-2 f(y)\right\| \leq c\left(\left.x\right|^{p}|y|^{q}\right.
$$

for all $x, y \in \mathbf{R} \backslash\{0\}$ such that $x \neq y$ and $x \neq-y$ then $f$ is a solution of the equation 1.1 on $\mathbf{R} \backslash\{0\}$. 
Proof. Observe that there exists $m_{0} \in \mathbf{N}_{0}$ such that :

$$
2 \sqrt[3]{m+1}^{p+q}+2 \sqrt[3]{m}^{p+q}+\sqrt[3]{2 m+1}^{p+q}<1
$$

for $m \geq m_{0}$.

Let us fix $m \in \mathbf{N}_{m_{0}}$. Replacing $x$ by $\sqrt[3]{m+1} x$ and $y$ by $\sqrt[3]{-m} x$ in inequality (2.8), we get :

$\|2 f(\sqrt[3]{m+1} x)+2 f(\sqrt[3]{-m} x)-f(\sqrt[3]{2 m+1} x)-f(x)\| \leq c \sqrt[3]{m+1} \sqrt[3]{m}^{q}|x|^{p+q}$,

for $x \in \mathbf{R} \backslash\{0\}$.

Further, we define operators $\mathcal{T}: X^{\mathbf{R}} \rightarrow X^{\mathbf{R}}$ and $\varepsilon: \mathbf{R} \rightarrow \mathbf{R}_{+}$by

$\mathcal{T} \xi(x)=2 \xi(\sqrt[3]{m+1} x)+2 \xi(\sqrt[3]{-m} x)-\xi(\sqrt[3]{2 m+1} x), \quad x \in \mathbf{R} \backslash\{0\}, \xi \in X^{\mathbf{R}}$

and

$$
\varepsilon(x)=c \sqrt[3]{m+1} \sqrt[p]{m^{q}}|x|^{p+q}, \quad x \in \mathbf{R} \backslash\{0\} .
$$

Inequality (2.9) become as the form :

$$
\|\mathcal{T} f(x)-f(x)\| \leq \varepsilon(x), x \in \mathbf{R} \backslash\{0\} .
$$

The operator :

$$
\Lambda \eta(x)=2 \eta(\sqrt[3]{m+1} x)+2 \eta(\sqrt[3]{-m} x)+\eta(\sqrt[3]{2 m+1} x)
$$

for all $\eta \in \mathbf{R}_{+}^{\mathbf{R}}, x \in \mathbf{R} \backslash\{0\}$, has the form described in $\left(H_{3}\right)$ with $k=3$ and

$f_{1}(x)=\sqrt[3]{m+1} x, f_{2}(x)=\sqrt[3]{-m} x, f_{3}(x)=\sqrt[3]{2 m+1} x$ and $L_{1}(x)=2$, $L_{2}(x)=2, L_{3}(x)=1$.

Moreover, for every $\xi, \mu \in X^{\mathbf{R} \backslash\{0\}}, x \in \mathbf{R} \backslash\{0\}$ :

$$
\begin{aligned}
\|\mathcal{T} \xi(x)-\mathcal{T} \mu(x)\| & =\| 2(\xi(\sqrt[3]{m+1} x)-\mu(\sqrt[3]{m+1} x))+2(\xi(\sqrt[3]{-m} x)-\mu(\sqrt[3]{-m} x)) \\
& -(\xi(\sqrt[3]{2 m+1} x)-\mu(\sqrt[3]{2 m+1} x)) \| \\
& \leq 2\|(\xi-\mu)(\sqrt[3]{m+1} x)\|+2\|(\xi-\mu)(\sqrt[3]{-m} x)\|+\|(\xi-\mu)(\sqrt[3]{2 m+1} x)\| \\
& =\sum_{i=1}^{3} L_{i}(x)\left\|(\xi-\mu) f_{i}(x)\right\| .
\end{aligned}
$$


As $2 \sqrt[3]{m+1}^{p+q}+2 \sqrt[3]{m}^{p+q}+\sqrt[3]{2 m+1}^{p+q}<1$, we have

$$
\begin{aligned}
& \varepsilon^{*}(x)=\sum_{n=0}^{\infty} \Lambda^{n} \epsilon(x) \\
& =\sum_{n=0}^{\infty} \Lambda^{n}\left(c \sqrt[3]{m+1}^{p} \sqrt[3]{m}^{q}|x|^{p+q}\right) \\
& =c \sqrt[3]{m+1} \sqrt[3]{m}^{q}|x|^{p} \sum_{n=0}^{\infty}\left(2 \sqrt[3]{m+1}^{p+q}+2 \sqrt[3]{m}^{p+q}+\sqrt[3]{2 m+1}^{p+q}\right)^{n} \\
& =\frac{c \sqrt[3]{m+1} p \sqrt[3]{m^{q}}|x|^{p+q}}{1-\left(2 \sqrt[3]{m+1} \sqrt[3]{p+q}^{p+2} \sqrt[3]{m}^{p+q}+\sqrt[3]{2 m+1}^{p+q}\right)}<\infty .
\end{aligned}
$$

According to theorem (1.1), there exists a fixed point $\mathcal{T}_{m}: \mathbf{R} \rightarrow X$ of the operator $\mathcal{T}$ such that:

$$
\left\|f(x)-\mathcal{T}_{m}(x)\right\| \leq \frac{c \sqrt[3]{m+1}^{p} \sqrt[3]{m}^{q}|x|^{p+q}}{1-\left(2 \sqrt[3]{m+1}^{p+q}+2 \sqrt[3]{m}^{p+q}+\sqrt[3]{2 m+1}^{p+q}\right)}
$$

Moreover $\mathcal{T}_{m}(x)=\lim _{n \rightarrow \infty} \mathcal{T}^{n}(f(x)), x \in \mathbf{R} \backslash\{0\}$. To prove that $\mathcal{T}_{m}$ is solution of (1.1), we will show that :

$$
\begin{aligned}
& \left\|\mathcal{T}^{n} f\left(\sqrt[3]{x^{3}+y^{3}}\right)+\mathcal{T}^{n} f\left(\sqrt[3]{x^{3}-y^{3}}\right)-2 \mathcal{T}^{n} f(x)-2 \mathcal{T}^{n} f(y)\right\| \\
& \quad \leq c\left(2 \sqrt[3]{m+1}^{p+q}+2 \sqrt[3]{m}^{p+q}+\sqrt[3]{2 m+1}^{p+q}\right)^{n}|x|^{p}|y|^{q}
\end{aligned}
$$

for $x, y \in \mathbf{R} \backslash\{0\}$ with $x \neq y, x \neq-y$ and $n \in \mathbf{N}_{0}$. Indeed, if $n=0$, then (2.13) is simply (2.8). So we take $n \in \mathbf{N}_{0}$ and suppose that (2.13) holds for $n$ and $x, y \in \mathbf{R} \backslash\{0\}$ with $x \neq y$ and $x \neq-y$, then 


$$
\begin{aligned}
& \left\|\mathcal{T}^{n+1} f\left(\sqrt[3]{x^{3}+y^{3}}\right)+\mathcal{T}^{n+1} f\left(\sqrt[3]{x^{3}-y^{3}}\right)-2 \mathcal{T}^{n+1} f(x)-2 \mathcal{T}^{n+1} f(y)\right\| \\
& =\left\|\mathcal{T}\left(\mathcal{T}^{n} f\left(\sqrt[3]{x^{3}+y^{3}}\right)\right)+\mathcal{T}\left(\mathcal{T}^{n} f\left(\sqrt[3]{x^{3}-y^{3}}\right)\right)-2 \mathcal{T}\left(\mathcal{T}^{n} f(x)\right)-2 \mathcal{T}\left(\mathcal{T}^{n} f(y)\right)\right\| \\
& =2 \mathcal{T}^{n} f\left(\sqrt[3]{m+1} \sqrt[3]{x^{3}+y^{3}}\right)+2 \mathcal{T}^{n} f\left(\sqrt[3]{-m} \sqrt[3]{x^{3}+y^{3}}\right)-\mathcal{T}^{n} f\left(\sqrt[3]{2 m+1} \sqrt[3]{x^{3}+y^{3}}\right) \\
& +2 \mathcal{T}^{n} f\left(\sqrt[3]{m+1} \sqrt[3]{x^{3}-y^{3}}\right)+2 \mathcal{T}^{n} f\left(\sqrt[3]{-m} \sqrt[3]{x^{3}-y^{3}}\right)-\mathcal{T}^{n} f\left(\sqrt[3]{2 m+1} \sqrt[3]{x^{3}-y^{3}}\right) \\
& -4 \mathcal{T}^{n} f(\sqrt[3]{m+1} x)-4 \mathcal{T}^{n} f(\sqrt[3]{-m} x)+2 \mathcal{T}^{n} f(\sqrt[3]{2 m+1} x) \\
& -4 \mathcal{T}^{n} f(\sqrt[3]{m+1} y)-4 \mathcal{T}^{n} f(\sqrt[3]{-m} y)+2 \mathcal{T}^{n} f(\sqrt[3]{2 m+1} y) \\
& =\| 2 \mathcal{T}^{n} f\left(\sqrt[3]{(\sqrt[3]{m+1} x)^{3}+(\sqrt[3]{m+1} y)^{3}}\right)+2 \mathcal{T}^{n} f\left(\sqrt[3]{(\sqrt[3]{-m} x)^{3}+(\sqrt[3]{-m} y)^{3}}\right) \\
& -\mathcal{T}^{n} f\left(\sqrt[3]{(\sqrt[3]{2 m+1} x)^{3}+(\sqrt[3]{2 m+1} y)^{3}}\right)+2 \mathcal{T}^{n} f\left(\sqrt[3]{(\sqrt[3]{m+1} x)^{3}-(\sqrt[3]{m+1} y)^{3}}\right) \\
& +2 \mathcal{T}^{n} f\left(\sqrt[3]{(\sqrt[3]{-m} x)^{3}-(\sqrt[3]{-m} y)^{3}}\right)-\mathcal{T}^{n} f\left(\sqrt[3]{(\sqrt[3]{2 m+1} x)^{3}-(\sqrt[3]{2 m+1} y)^{3}}\right) \\
& -4 \mathcal{T}^{n} f(\sqrt[3]{m+1} x)-4 \mathcal{T}^{n} f(\sqrt[3]{-m} x)+2 \mathcal{T}^{n} f(\sqrt[3]{2 m+1} x) \\
& -4 \mathcal{T}^{n} f(\sqrt[3]{m+1} y)-4 \mathcal{T}^{n} f(\sqrt[3]{-m} y)+2 \mathcal{T}^{n} f(\sqrt[3]{2 m+1} y) \\
& \leq 2 \| \mathcal{T}^{n} f\left(\sqrt[3]{(\sqrt[3]{m+1} x)^{3}+(\sqrt[3]{m+1} y)^{3}}\right)+\mathcal{T}^{n} f\left(\sqrt[3]{(\sqrt[3]{m+1} x)^{3}-(\sqrt[3]{m+1} y)^{3}}\right) \\
& -2 \mathcal{T}^{n} f(\sqrt[3]{m+1} x)-2 \mathcal{T}^{n} f(\sqrt[3]{m+1} y) \| \\
& +2 \| \mathcal{T}^{n} f\left(\sqrt[3]{(\sqrt[3]{-m} x)^{3}+(\sqrt[3]{-m} y)^{3}}\right)+\mathcal{T}^{n} f\left(\sqrt[3]{(\sqrt[3]{-m} x)^{3}-(\sqrt[3]{-m} y)^{3}}\right) \\
& -2 \mathcal{T}^{n} f(\sqrt[3]{-m} x)-2 \mathcal{T}^{n} f(\sqrt[3]{-m} y) \\
& +\| \mathcal{T}^{n} f\left(\sqrt[3]{(\sqrt[3]{2 m+1} x)^{3}+(\sqrt[3]{2 m+1} y)^{3}}\right)+\mathcal{T}^{n} f\left(\sqrt[3]{(\sqrt[3]{2 m+1} x)^{3}-(\sqrt[3]{2 m+1} y)^{3}}\right) \\
& -2 \mathcal{T}^{n} f(\sqrt[3]{2 m+1} x)-2 \mathcal{T}^{n} f(\sqrt[3]{2 m+1} y) \| \\
& \leq c\left(2 \sqrt[3]{m+1}^{p+q}+2 \sqrt[3]{m}^{p+q}+\sqrt[3]{2 m+1}^{p+q}\right)^{n}\left(\left(2 \sqrt[3]{m+1}^{p+q}\right)|x|^{p}|y|^{q}\right) \\
& +c\left(2 \sqrt[3]{m+1}^{p+q}+2 \sqrt[3]{m}^{p+q}+\sqrt[3]{2 m+1}^{p+q}\right)^{n}\left(2 \sqrt[3]{m}^{p+q}|x|^{p}|y|^{q}\right) \\
& +c\left(2 \sqrt[3]{m+1}^{p+q}+2 \sqrt[3]{m}^{p+q}+\sqrt[3]{2 m+1}^{p+q}\right)^{n}\left(\sqrt[3]{2 m+1}^{p+q}|x|^{p}|y|^{q}\right) \\
& \leq c\left(2 \sqrt[3]{m+1}^{p+q}+2 \sqrt[3]{m}^{p+q}+\sqrt[3]{2 m+1}^{p+q}\right)^{n+1}|x|^{p}|y|^{q} .
\end{aligned}
$$

By induction, we show that (2.13) holds for all $x, y \in \mathbf{R} \backslash\{0\}$ with $x \neq y, x \neq-y$ and $n \in \mathbf{N}_{0}$. Letting $n \rightarrow \infty$ in (2.13), we obtain :

$\mathcal{T}_{m}\left(\sqrt[3]{x^{3}+y^{3}}\right)+\mathcal{T}_{m}\left(\sqrt[3]{x^{3}-y^{3}}\right)=2 \mathcal{T}_{m}(x)+2 \mathcal{T}_{m}(y) \quad x, y \in \mathbf{R} \backslash\{0\}, x \neq-y, x \neq y$

We prove now that $\mathcal{T}_{m}$ is the unique operator solution of (1.1) and satisfies the condition (2.12) for all $x \in \mathbf{R} \backslash\{0\}$.

So, let $\mathcal{T}_{0}: \mathbf{R} \rightarrow X$ an other solution of (1.1) such that : 


$$
\left\|f(x)-\mathcal{T}_{0}(x)\right\| \leq \frac{c \sqrt[3]{m+1} \sqrt[3]{m}^{q}|x|^{p+q}}{1-\left(2 \sqrt[3]{m+1}^{p+q}+2 \sqrt[3]{m}^{p+q}+\sqrt[3]{2 m+1}^{p+q}\right)} \quad, x \in \mathbf{R} \backslash\{0\}
$$

then for every $x \in \mathbf{R} \backslash\{0\}$ :

$$
\begin{aligned}
\left\|\mathcal{T}_{m}(x)-\mathcal{T}_{0}(x)\right\| & \leq\left\|\mathcal{T}_{m}(x)-f(x)\right\|+\left\|\mathcal{T}_{0}(x)-f(x)\right\| \\
& \left.\leq \frac{2 c \sqrt[3]{m+1}^{p} \sqrt[3]{m}^{q}|x|^{p+q}}{1-\left(2 \sqrt[3]{m+1}^{p+q}+2 \sqrt[3]{m}^{p+q}+\sqrt[3]{2 m+1} p+q\right.}\right)
\end{aligned}
$$

putting

$$
\alpha=2 c \sqrt[3]{m+1} \sqrt[3]{m^{q}}
$$

and show that,

$$
\left\|\mathcal{T}_{m}(x)-\mathcal{T}_{0}(x)\right\| \leq \alpha|x|^{p+q} \sum_{n=j}^{n=\infty}\left(2 \sqrt[3]{m+1}^{p+q}+2 \sqrt[3]{m}^{p+q}+\sqrt[3]{2 m+1}^{p+q}\right)^{n}
$$

for each $j \in \mathbf{N}_{0}$ and $x \in \mathbf{R} \backslash\{0\}$.

For $j=0$ inequality (2.15) is exactly (2.14). So we fix $l \in \mathbf{N}_{0}$ and assume that (2.15) holds for $j=l$, then

$$
\begin{aligned}
& \left\|\mathcal{T}_{m}(x)-\mathcal{T}_{0}(x)\right\|=\| 2 \mathcal{T}_{m}(\sqrt[3]{m+1} x)+2 \mathcal{T}_{m}(\sqrt[3]{-m} x)-\mathcal{T}_{m}(\sqrt[3]{2 m+1} x) \\
& -2 \mathcal{T}_{0}(\sqrt[3]{m+1} x)-2 \mathcal{T}_{0}(\sqrt[3]{-m} x)+\mathcal{T}_{0}(\sqrt[3]{2 m+1} x) \| \\
& \leq 2\left\|\mathcal{T}_{m}(\sqrt[3]{m+1} x)-\mathcal{T}_{0}(\sqrt[3]{m+1} x)\right\|+2\left\|\mathcal{T}_{m}(\sqrt[3]{-m} x)-\mathcal{T}_{0}(\sqrt[3]{-m} x)\right\| \\
& +\left\|\mathcal{T}_{m}(\sqrt[3]{2 m+1} x)-\mathcal{T}_{0}(\sqrt[3]{2 m+1} x)\right\| \\
& \leq \alpha\left(2 \sqrt[3]{m+1}^{p+q}+2 \sqrt[3]{m}^{p+q}+\sqrt[3]{2 m+1} p+q\right)|x|^{p+q} \sum_{n=l}^{n=\infty} \\
& \left(2 \sqrt[3]{m+1}^{p+q}+2 \sqrt[3]{m} p+q+\sqrt[3]{2 m+1} p+\right)^{n} \\
& =\alpha|x|^{p+q} \sum_{n=l+1}^{n=\infty}\left(2 \sqrt[3]{m+1}^{p+q}+2 \sqrt[3]{m}^{p+q}+\sqrt[3]{2 m+1}^{p+q}\right)^{n}, \quad x \in \mathbf{R} \backslash\{0\} .
\end{aligned}
$$

Thus, we have shown that (2.15) holds true for every $j \in \mathbf{N}_{0}$.

Now letting $j \rightarrow \infty$ in $(2.15)$ we get $\mathcal{T}_{m}=\mathcal{T}_{0}$.

Theorem 2.5. Let $X$ be a Banach space, $c \geq 0$ and $p, q \in \mathbf{R}$ such that $p+q>6$. If $f: \mathbf{R} \rightarrow X$ satisfies : the equation (2.8) then $f$ is a solution of the equation (1.1) on $\mathbf{R} \backslash\{0\}$. 
Proof. By a similar method of the previous theorem with replacing $x$ by $\sqrt[3]{\frac{m+1}{2 m}} x$ and $y$ by $\sqrt[3]{\frac{m-1}{2 m}} x$ we can easily prove this theorem.

\section{Consequences}

We finish this paper with some corollaries concerning the inhomogeneous of the cubic radical functional equation (1.1) in two case of the hyperstability.

Corollary 3.1. Let $X$ be a Banach space, $G: \mathbf{R} \backslash\{0\} \times \mathbf{R} \backslash\{0\} \rightarrow X$ such that $G(x, y) \neq 0$ for some $x, y \in \mathbf{R} \backslash\{0\}$ with $x \neq y$ and $x \neq-y$. Assume that $p$ satisfy $p<0$ or $p>6$ and $c>0$ such that

$$
\|G(x, y)\| \leq c\left(|x|^{p}+|y|^{p}\right), \quad x, y \in \mathbf{R} \backslash\{0\}, x \neq y, x \neq-y
$$

Then the functional equation

$g\left(\sqrt[3]{x^{3}+y^{3}}\right)+g\left(\sqrt[3]{x^{3}-y^{3}}\right)=2 g(x)+2 g(y)+G(x, y), \quad x, y \in \mathbf{R} \backslash\{0\}, x \neq y, x \neq-y$

has no solution in the class of functions $g: \mathbf{R} \backslash\{0\} \rightarrow X$.

Proof. Suppose that $g: \mathbf{R} \backslash\{0\} \rightarrow X$ solution of the equation (3.1). Define $g: \mathbf{R} \rightarrow X$ by $f(x)=g(x)$ for $x \in \mathbf{R} \backslash\{0\}$ and $f(0)=0$. Then

$$
\left\|f\left(\sqrt[3]{x^{3}+y^{3}}\right)+f\left(\sqrt[3]{x^{3}-y^{3}}\right)-2 f(x)-2 f(y)\right\|=\|G(x, y)\| \leq c\left(\|x\|^{p}+\|y\|^{p}\right)
$$

Consequently $f$ is a solution of the equation (1.1), which means that $G(x, y) \geq 0$ for some $x, y \in \mathbf{R} \backslash\{0\}$. This is a contradiction.

Corollary 3.2. Let $X$ be a Banach space, $G: \mathbf{R} \backslash\{0\} \times \mathbf{R} \backslash\{0\} \rightarrow X$ such that $G(x, y) \neq 0$ for some $x, y \in \mathbf{R} \backslash\{0\}$ with $x \neq y$ and $x \neq-y$. Assume that $p, q$ satisfy $p+q<0$ or $p+q>6$ and $C>0$ such that

$$
\|G(x, y)\| \leq c|x|^{p}|y|^{q}, \quad x, y \in \mathbf{R} \backslash\{0\}, x \neq y, x \neq-y .
$$

Then the functional equation

$g\left(\sqrt[3]{x^{3}+y^{3}}\right)+g\left(\sqrt[3]{x^{3}-y^{3}}\right)=2 g(x)+2 g(y)+G(x, y), \quad x, y \in \mathbf{R} \backslash\{0\}, x \neq y, x \neq-y$, has no solution in the class of functions $g: \mathbf{R} \backslash\{0\} \rightarrow X$. 


\section{References}

[1] L. Aiemsomboon and W. Sintunavarat, "On generalized hyperstability of a general linear equation", Acta mathematica hungarica, vol. 149, no. 2, pp. 413-422, Aug. 2016, doi: 10.1007/s10474-016-0621-2.

[2] M. Almahalebi, A. Charifi and S. Kabbaj, "Hyperstability of a Cauchy functional equation", Journal of nonlinear analysis and optimization: theory \& applications, vol. 6, no. 2, pp. 127-137, 2015. [On line]. Available: https://bitly/207wqZf

[3] T. Aoki, "On the stability of the linear transformation in Banach spaces", Journal of the mathematical society of Japan, vol. 2, no. 1-2, pp. 64-66, 1950, doi: 10.2969/jmsj/00210064.

[4] D. G. Bourgin, "Classes of transformations and bordering transformations", Bulletin of the American mathematical society, vol. 57, no. 4, pp. 223-237, 1951, doi: 10.1090/S0002-9904-1951-09511-7.

[5] J.BrzdękandJ.Tabor, “A note on stability of additive mappings", in Stability of mappings of Hyers-Ulam type, T.M. Rassias, Ed. Palm Harbor, FL: Hadronic Press, 1994, pp. 19-22.

[6] J. Brzdęk, J. Chudziak, and Z. Páles, "A fixed point approach to stability of functional equations", Nonlinear analysis: theory, methods \& applications, vol. 74, no. 17, pp. 6728-6732, Dec. 2011, doi: 10.1016/j.na.2011.06.052.

[7] J.Brzdęk,Hyperstability of the Cauchy equation on restricted domains,Acta mathematica hungarica, vol.141, no.1-2, pp.58-67,0ct. 2013, doi: 10.1007/s10474-013-0302-3.

[8] J. Brzdęk, "Remark 3. 16th International Conference on Functional Equations and Inequalities, Będlewo, Poland, May 17-23, 2015", Annales universitatis paedagogicae cracoviensis. Studia mathematica, vol. 14, no. 1, p. 196, Dec. 2015, doi: 10.1515/aupcsm-2015-0012.

[9] M. Eshaghi Gordji, H. Khodaei, A. Ebadian, and G. H. Kim, "Nearly radical quadratic functional equations in p-2-normed spaces", Abstract and applied analysis, Art. ID. 896032, 2012, doi: 10.1155/2012/896032.

[10] D. H. Hyers, "On the stability of the linear functional equation", Proceedings of the National Academy of Sciences of the United States of America, vol. 27, no. 4 pp. 222-224, Apr. 1941, doi: 10.1073/pnas.27.4.222.

[11] H. Khodaei, M. Eshaghi Gordji, S. S. Kim, and Y. J. Cho, "Approximation of radical functional equations related to quadratic and quartic mappings", Journal of mathematical analysis and applications, vol. 395, no.1, pp. 284-297, Nov. 2012, doi: 10.1016/j.jmaa.2012.04.086. 
[12] S. S. Kim, Y. J. Cho and M. Eshaghi Gordji, "On the generalized Hyers-UlamRassias stability problem of radical functional equations", Journal of inequalities and applications, Art. ID. 186, Aug. 2012, doi: 10.1186/1029-242X2012-186.

[13] G. Maksa and Z. Pales, "Hyperstability of a class of linear functional equations", Acta mathematica academiae paedagogicae nyiregyhaziensis, vol. 17, no. 2, pp. 107-112, 2001. [On line]. Available: https://bit.ly/2RzfnBt

[14] Th. M. Rassias, "On the stability of the linear mapping in Banach spaces", Proceeding of the American mathematical society, vol. 72, no. 1, pp. 297-300, 1978, doi: 10.1090/S0002-9939-1978-0507327-1.

[15] Th. M. Rassias, "Problem 16, $2^{\circ}$. The Twenty-seventh International Symposium on Functional Equations, August 14-24, 1989, Bielsko-BiałKatowice-Kraków, Poland", Aequationes mathematicae, vol. 39, no. 2-3, pp. 292-293, Apr. 1990, doi: 10.1007/BF01833155.

[16] Th. M. Rassias, "On a modified Hyers-Ulam sequence”, Journal of mathematical analysis and applications, vol. 158, no. 1, pp. 106-113, Jun. 1991, doi: 10.1016/0022-247X(91)90270-A.

[17] S. M. Ulam, Problems in modern mathematics. New York, NY: John Wiley \& Sons, 1964. 